\title{
Asymmetric Deviation of the Scattering Cross Section around Ly $\alpha$ by Atomic Hydrogen
}

\author{
Hee-Won Lee \\ Department of Astronomy and Space Sciences, Sejong University, Seoul, 143-747, Korea \\ hwlee@sejong.ac.kr
}

\begin{abstract}
We investigate the asymmetry of the scattering cross section of radiation around Ly $\alpha$ by atomic hydrogen, which may be applied to analyses of scattering media with high column neutral hydrogen densities including damped Ly $\alpha$ absorption systems of quasars. The exact scattering cross section is given by the Kramers-Heisenberg formula obtained from the fully quantum mechanical second-order time dependent theory, where, in the case of hydrogen, each matrix element is given in a closed analytical form. The asymmetric deviation of the scattering cross section from the Lorentzian near the line center is computed by expanding the Kramers-Heisenberg formula in terms of $\Delta \omega / \omega_{L y \alpha}$, where $\omega_{L y \alpha}$ is the angular frequency of the Ly $\alpha$ transition and $\Delta \omega$ is the deviation of incident radiation from $\omega_{L y \alpha}$. To the first order of $\Delta \omega / \omega_{L y \alpha}$, we obtain $\sigma(\omega)=\sigma_{T}\left(0.5 f_{12} \omega_{L y \alpha} / \Delta \omega\right)^{2}\left(1-1.79 \Delta \omega / \omega_{L y \alpha}\right)$, where $\sigma_{T}$ is the Thomson scattering cross section and $f_{12}=0.4162$ is the oscillator strength for the Ly $\alpha$ transition. With this deviation, the line center of the damped wing profile apparently shifts blueward of the true Ly $\alpha$ line center. In the case of a damped Ly $\alpha$ system with a $\mathrm{H}$ I column density $5 \times 10^{21} \mathrm{~cm}^{-2}$, the apparent line center shift relative to the true center amounts to $0.2 \AA$ resulting in an underestimation of redshift by $\Delta z \sim 10^{-4}$. A measurable underestimation by an amount of $\Delta z \sim 10^{-3}$ is expected for absorbing systems with $N_{H I} \geq 4 \times 10^{22} \mathrm{~cm}^{-2}$.
\end{abstract}

Subject headings: atomic physics — radiative transfer — cosmology — quasar

\section{Introduction}

The scattering process of electromagnetic radiation by an atom is regarded as a secondorder quantum mechanical process involving the annihilation of an incident photon and the 
creation of a scattered photon. Therefore, from the second-order time dependent perturbation theory, we can obtain the scattering cross section, which is known as the KramersHeisenberg formula (e.g. Sakurai 1969). The exact scattering cross section can be obtained after summing all bound state contributions and integrating over all continuum state contributions related with the dipole processes of annihilation and creation of photons. Hydrogen being a single electron atom, the energy eigenstate wavefunctions of hydrogen are analytically known and hence each matrix element appearing in the Kramers-Heisenberg formula is given in a closed form.

Near resonance, the scattering cross section is excellently approximated by the Lorentzian characterized by the narrow radiation damping term that can be interpreted as the life time of the excited state. The Lorentzian dependence is obtained since the cross section is dominated by the single contribution from the excited state relevant to the resonance whereas the contributions from the other states are almost completely negligible. This Lorentzian approximation is quite generally used in the astronomical community for analyses of resonance lines.

Far from resonance, the radiation damping term is negligibly important and the scattering cross section is approximately proportional to $\Delta \omega^{-2}$, where $\Delta \omega$ is the difference of the angular frequency of the incident radiation and that of the Ly $\alpha$ line center, $\omega_{L y \alpha}$. This cross section given by the Lorentzian is symmetric with respect to the line center in frequency space. However, the true scattering cross section deviates from $\Delta \omega^{-2}$ very far from the line center as the contributions increase from the other energy eigenstates including both bound and continuum states. The zeroth order term $\sigma \propto\left(\omega_{L y \alpha} / \Delta \omega\right)^{2}$ being symmetric with respect to the line center in frequency space, the first order term $\Delta \omega / \omega_{L y \alpha}$ for this deviation will contribute to the asymmetry of the scattering cross section. Therefore, a very careful quantitative consideration of this effect should be taken in order to obtain an accurate estimation of the true line center of high H I column density absorption systems.

H I absorption systems are easily found in typical quasar spectra and are very useful to investigate the baryonic matter content and the large scale structure in the universe. The quasar H I absorption systems include the Ly $\alpha$ forest, Lyman limit systems and damped Ly $\alpha$ systems, which are classified according to the $\mathrm{H}$ I column density $N_{H I}$. A number of damped Ly $\alpha$ systems (DLA's) are known to exhibit H I column density in excess of $10^{21} \mathrm{~cm}^{-2}$ (e.g. Turnshek \& Rao, 1998). Very high H I column density media may be found in the early universe, when the reionization is not complete and the universe is only partially ionized by the first objects (Gunn \& Peterson 1965, Scheuer 1965). In these high column $\mathrm{H}$ I density media, the correct redshift of the absorbing system can be obtained after proper considerations of the asymmetric scattering cross section in frequency space. 
In this paper, we calculate the asymmetric deviation of the scattering cross section of radiation around Ly $\alpha$ by atomic hydrogen and apply our results to high $\mathrm{H}$ I column density scattering media that may be important in observational cosmology.

\section{Calculation}

\subsection{The Kramers-Heisenberg Formula}

We start with the Kramers-Heisenberg formula that is obtained from the fully quantum mechanical second-order time dependent theory (e.g. Sakurai 1969, Merzbacher 1970). In

terms of the matrix elements of the dipole operator $(e \mathbf{x})$, the differential cross section $\frac{d \sigma}{d \Omega}$ for incident radiation with angular frequency $\omega$ is given by the Kramers-Heisenberg formula, which can be written as

$$
\begin{aligned}
\frac{d \sigma}{d \Omega} & =\frac{r_{0}^{2} m_{e}^{2}}{\hbar^{2}} \mid \sum_{n} \omega \omega_{n 1}\left(\frac{\left(\mathbf{x} \cdot \epsilon^{\left(\alpha^{\prime}\right)}\right)_{1 n}\left(\mathbf{x} \cdot \epsilon^{(\alpha)}\right)_{n 1}}{\omega_{n 1}-\omega}\right. \\
& \left.-\frac{\left(\mathbf{x} \cdot \epsilon^{(\alpha)}\right)_{1 n}\left(\mathbf{x} \cdot \epsilon^{\left(\alpha^{\prime}\right)}\right)_{n 1}}{\omega_{n 1}+\omega}\right) \\
& +\int_{0}^{\infty} d n^{\prime} \omega \omega_{n^{\prime} 1}\left(\frac{\left(\mathbf{x} \cdot \epsilon^{\left(\alpha^{\prime}\right)}\right)_{1 n^{\prime}}\left(\mathbf{x} \cdot \epsilon^{(\alpha)}\right)_{n^{\prime} 1}}{\omega_{n^{\prime} 1}-\omega}\right. \\
& \left.-\frac{\left(\mathbf{x} \cdot \epsilon^{(\alpha)}\right)_{1 n^{\prime}}\left(\mathbf{x} \cdot \epsilon^{\left(\alpha^{\prime}\right)}\right)_{n^{\prime} 1}}{\omega_{n^{\prime} 1}+\omega}\right)\left.\right|^{2} .
\end{aligned}
$$

where $m_{e}$ is the electron mass, $r_{0}=e^{2} / m_{e} c^{2}$ is the classical electron radius, $\left(\mathbf{x} \cdot \epsilon^{\left(\alpha^{\prime}\right)}\right)_{1 n}$ represents the matrix element of the position operator between the ground $1 s$ state and excited $n p$ state, and $\epsilon^{\alpha}, \epsilon^{\alpha^{\prime}}$ are polarization vectors of incident and scattered radiation, respectively. Here, $\omega_{n 1}$ is the angular frequency between the bound $n p$ state and the ground $1 s$ state, and $\omega_{n^{\prime} 1}$ is the angular frequency of the transition between $1 s$ and the continuum $n^{\prime} p$ state. In the atomic units adopted in this work, the bound $n p$ state has an energy eigenvalue $E_{n}=-\frac{1}{2 n^{2}}$ and correspondingly $\omega_{n 1}=\left(\frac{1}{2}\right)\left(1-1 / n^{2}\right)$. Similarly, $E_{n^{\prime}}=\frac{1}{2\left(n^{\prime}\right)^{2}}$ and $\omega_{n^{\prime} 1}=\left(\frac{1}{2}\right)\left(1+1 / n^{2}\right)$.

In the limit $\omega \rightarrow \infty$, with the use of the closure relation it can be shown that the Kramers-Heisenberg formula yields a constant scattering cross section independent of the wavelength of incident radiation, which is the Thomson scattering cross section $d \sigma / d \Omega=$ $r_{0}^{2}\left|\epsilon^{(\alpha)} \cdot \epsilon^{\left(\alpha^{\prime}\right)}\right|^{2}$. In the opposite limit where $\omega \ll \omega_{21}=\omega_{L y \alpha}$, another use of the completeness 
of the intermediate states gives

$$
\begin{aligned}
\frac{d \sigma}{d \Omega} & =\left(\frac{r_{0} m_{e}}{\hbar}\right)^{2} \omega^{4} \mid \sum_{I}\left(\frac{1}{\omega_{I 1}}\right)\left[\left(\mathbf{x} \cdot \epsilon^{\left(\alpha^{\prime}\right)}\right)_{1 I}\left(\mathbf{x} \cdot \epsilon^{(\alpha)}\right)_{I 1}\right. \\
& \left.+\left(\mathbf{x} \cdot \epsilon^{(\alpha)}\right)_{1 I}\left(\mathbf{x} \cdot \epsilon^{\left(\alpha^{\prime}\right)}\right)_{I 1}\right]\left.\right|^{2}
\end{aligned}
$$

which shows the well-known $\omega^{4}$ dependence of the Rayleigh scattering cross section in the low energy limit.

In order to investigate the behavior of the cross section around Ly $\alpha$, we introduce

$$
\Delta \omega \equiv \omega-\omega_{21}
$$

and expand the Kramers-Heisenberg formula in terms of $\Delta \omega / \omega_{21}$.

In the first term inside the summation of Eq. 1 with $n=2$, we can write

$$
\frac{\omega \omega_{21}}{\omega_{21}-\omega}=-\omega_{21}\left(1+\frac{\omega_{21}}{\Delta \omega}\right)
$$

In the same term for $n \geq 3$, we may write

$$
\frac{\omega}{\omega_{n 1}-\omega}=\frac{\omega_{21}}{\omega_{n 1}-\omega_{21}}\left[1+\frac{\omega_{n 1}}{\omega_{21}} \sum_{k=1}^{\infty}\left(\frac{\Delta \omega}{\omega_{n 1}-\omega_{21}}\right)^{k}\right] .
$$

In a similar way, for $n \geq 2$, the second term in the summation in Eq. 1 can be written as

$$
\frac{\omega}{\omega_{n 1}+\omega}=\frac{\omega_{21}}{\omega_{n 1}+\omega_{21}}\left[1-\frac{\omega_{n 1}}{\omega_{21}} \sum_{k=1}^{\infty}\left(\frac{-\Delta \omega}{\omega_{n 1}+\omega_{21}}\right)^{k}\right] .
$$

Similar expressions for continuum states are obtained in a straightforward manner.

Making use of the Wigner-Eckart theorem we may separate the matrix elements of the position operator into the angular part and the radial part, where the radial part is given by the reduced matrix elements $<f\|x\| i>$. (e.g. Merzbacher 1970). The angular part gives the same dipole type angular distribution as that for Thomson scattering or classical Rayleigh scattering (e.g. Ahn, Lee \& Lee 2000, Lee 1997, Schmid 1989).

Substituting Eqs 5,6 into Eq. 1, we obtain

$$
\begin{aligned}
\sigma & =\sigma_{T}\left(\frac{\omega_{21}}{\Delta \omega}\right)^{2} \mid \omega_{21}<x>_{12}^{2}+\omega_{21}<x>_{12}^{2}\left(\frac{\Delta \omega}{\omega_{21}}\right) \\
& -\sum_{n=3}^{\infty} \frac{\Delta \omega \omega_{n 1}}{\omega_{n 1}-\omega_{21}}\left[1+\frac{\omega_{n 1}}{\omega_{21}} \sum_{k=1}^{\infty}\left(\frac{\Delta \omega}{\omega_{n 1}-\omega_{21}}\right)^{k}\right]<x>_{n 1}^{2}
\end{aligned}
$$




$$
\begin{aligned}
& +\sum_{n=2}^{\infty} \frac{\Delta \omega \omega_{n 1}}{\omega_{n 1}+\omega_{21}}\left[1-\frac{\omega_{n 1}}{\omega_{21}} \sum_{k=1}^{\infty}\left(\frac{-\Delta \omega}{\omega_{n 1}+\omega_{21}}\right)^{k}\right]<x>_{n 1}^{2} \\
& -\int_{0}^{\infty} d n^{\prime} \frac{\Delta \omega \omega_{n^{\prime} 1}}{\omega_{n^{\prime} 1}-\omega_{21}}\left[1+\frac{\omega_{n^{\prime} 1}}{\omega_{21}} \sum_{k=1}^{\infty}\left(\frac{\Delta \omega}{\omega_{n^{\prime} 1}-\omega_{21}}\right)^{k}\right]<x>_{n^{\prime} 1}^{2} \\
& +\int_{0}^{\infty} d n^{\prime} \frac{\Delta \omega \omega_{n^{\prime} 1}}{\omega_{n^{\prime} 1}+\omega_{21}}\left[1-\frac{\omega_{n^{\prime} 1}}{\omega_{21}} \sum_{k=1}^{\infty}\left(\frac{-\Delta \omega}{\omega_{n^{\prime} 1}+\omega_{21}}\right)^{k}\right]<x>\left._{n^{\prime} 1}^{2}\right|^{2}
\end{aligned}
$$

where the atomic units have been used and angular integration has been performed. Here, the Thomson scattering cross section $\sigma_{T}=8 \pi r_{0}^{2} / 3=0.665 \times 10^{-24} \mathrm{~cm}^{2}$. The matrix elements of the dipole operators $\langle x\rangle_{n 1},\langle x\rangle_{n^{\prime}, 1}$ are easily found in textbooks on quantum mechanics (e.g. Berestetski, Lifshitz \& Pitaevskii 1971, Saslow \& Mills 1969, Bethe \& Salpeter 1967). The matrix elements are given by

$$
<x>_{n 1}=<1 s|x| n p>=\left[\frac{2^{8} n^{7}(n-1)^{2 n-5}}{3(n+1)^{2 n+5}}\right]^{\frac{1}{2}} a_{0}
$$

for the bound states. Here, the Bohr radius $a_{0}=\hbar^{2} / m e^{2}$ is set equal to 1 in atomic units. For the continuum states, the corresponding values are given by

$$
<x>_{n^{\prime} 1}=<1 s|x| n^{\prime} p>=\left[\frac{2^{8}\left(n^{\prime}\right)^{7} \exp \left[-4 n^{\prime} \tan ^{-1}\left(1 / n^{\prime}\right)\right]}{3\left[\left(n^{\prime}\right)^{2}+1\right]^{5}\left[1-\exp \left(-2 \pi n^{\prime}\right)\right]}\right]^{\frac{1}{2}}
$$

where we also adopt the atomic units.

Eq. 7 can be written as

$$
\sigma=\sigma_{T}\left(\frac{\omega_{21}}{\Delta \omega}\right)^{2}\left|A_{0}+A_{1}\left(\frac{\Delta \omega}{\omega_{21}}\right)+A_{2}\left(\frac{\Delta \omega}{\omega_{21}}\right)^{2}+\cdots\right|^{2}
$$

where the coefficients $A_{k}$ are obtained through the following relations

$$
\begin{aligned}
A_{0} & =\omega_{21}<x>_{12}^{2}=\frac{1}{2} f_{12}=0.2081 \\
A_{1} & =\frac{3}{2} \omega_{21}<x>_{12}^{2}-\frac{1}{2} \sum_{n=3}^{\infty} \frac{\omega_{21}^{2} \omega_{n 1}}{\omega_{n 1}^{2}-\omega_{21}^{2}}<x>_{1 n}^{2}= \\
& -\frac{1}{2} \int_{0}^{\infty} d n^{\prime} \frac{\omega_{21}^{2} \omega_{n^{\prime} 1}}{\omega_{n^{\prime} 1}^{2}-\omega_{21}^{2}}<x>_{n 1^{\prime}}^{2}=-0.1865 \\
A_{k} & =\left(\frac{-1}{2}\right)^{k} \omega_{21}<x>_{12}^{2}
\end{aligned}
$$




$$
\begin{aligned}
& -\sum_{n=3}^{\infty} \frac{\omega_{n 1}^{2}}{\omega_{21}}\left[\left(\frac{\omega_{21}}{\omega_{n 1}-\omega_{21}}\right)^{k}-\left(\frac{-\omega_{21}}{\omega_{n 1}+\omega_{21}}\right)^{k}\right]<x>_{n 1}^{2} \\
& -\int_{0}^{\infty} d n^{\prime} \frac{\omega_{n^{\prime} 1}^{2}}{\omega_{21}}\left[\left(\frac{\omega_{21}}{\omega_{n^{\prime} 1}-\omega_{21}}\right)^{k}-\left(\frac{-\omega_{21}}{\omega_{n^{\prime} 1}+\omega_{21}}\right)^{k}\right]<x>_{n^{\prime} 1}^{2} .
\end{aligned}
$$

Here, $f_{12}=0.4162$ is the oscillator strength for the Ly $\alpha$ transition.

The computation of the coefficients can be done in a straightforward way, from which we obtain a list of these coefficients up to $k=5$

$$
\begin{aligned}
& A_{1} / A_{0}=-0.8961 \\
& A_{2} / A_{0}=-1.222 \times 10^{1} \\
& A_{3} / A_{0}=-5.252 \times 10^{1} \\
& A_{4} / A_{0}=-2.438 \times 10^{2} \\
& A_{5} / A_{0}=-1.210 \times 10^{3} .
\end{aligned}
$$

Therefore, up to the first order in $\Delta \omega / \omega_{21}$, the scattering cross section of radiation around Ly $\alpha$

$$
\begin{aligned}
\sigma(\omega) & \simeq \sigma_{T}\left(\frac{f_{12}}{2}\right)^{2}\left(\frac{\omega_{21}}{\Delta \omega}-0.8961\right)^{2} \\
& \simeq \sigma_{T}\left(\frac{f_{12}}{2}\right)^{2}\left(\frac{\omega_{21}}{\Delta \omega}\right)^{2}\left(1-1.792 \frac{\Delta \omega}{\omega_{21}}\right)
\end{aligned}
$$

In Fig. 1, we show the scattering cross section multiplied by a column density $N_{H I}=$ $4 \times 10^{22} \mathrm{~cm}^{-2}$, that is the scattering optical depth of a neutral medium with this column

density. The solid line represents the result obtained from the Kramers-Heisenberg formula, the dotted line depicts the first order approximation to it obtained in Eq. 13 and the dashed line shows the zeroth approximation proportional to $\Delta \omega^{-2}$ obtained from the Lorentzian by ignoring the damping part. The inclusion of the first order term implies the introduction of asymmetry of the scattering cross section with respect to the line center in frequency space. As is apparent in Fig. 1, the scattering cross section is smaller than the Lorentzian blueward of the line center, whereas it is larger in the opposite part.

\subsection{Applications to High $N_{H I}$ Media}

In Fig. 2 we show the absorption profile $e^{-\tau(\lambda)}$ that is expected from a damped Ly $\alpha$ system with a column density $N_{H I}=5 \times 10^{21} \mathrm{~cm}^{-2}$. The thick solid line represents the 
result obtained using the first-order corrected formula and the dotted line is the absorption profile obtained using the Voigt profile by shifting the center wavelength by an amount $\Delta \lambda=0.187 \AA$ to the blue part. The wavelength shift by this amount is determined using the least square method that best fits the thick solid line. Because in the observational data it is difficult to obtain the exact continuum level, we minimize the difference $\left|e^{-\tau_{L}(\lambda)}-e^{-\tau_{L C}(\lambda)}\right|^{2}$ in the wavelength interval between $1180 \AA$ and $1250 \AA$, where the suppression of the continuum is larger than 20 percent.

Therefore, if we apply the Voigt profile fitting process to observational data for an $\mathrm{H}$ I scattering system with $N_{H I}=5 \times 10^{21} \mathrm{~cm}^{-2}$, we may underestimate the redshift of the H I system by an amount of $\Delta z=0.187 / 1215.67=1.54 \times 10^{-4}$ or in terms of the velocity scale $\Delta v=39 \mathrm{~km} \mathrm{~s}^{-1}$.

Rao \& Turnshek (2000) investigated the damped Ly $\alpha$ absorption system along the line of sight to the quasar PKS 1127-145, which was best fitted with the Voigt profile with $z=0.3127$ (see also Rao et al. 2003). Hence, the first order correction from our quantum mechanical consideration may affect the fourth decimal place of $z$, which is not critically important considering the accuracy of $\Delta z=0.001$ quoted by the authors. Among DLA's so far investigated, the highest H I column density is $\sim N_{\mid H I}=5 \times 10^{21} \mathrm{~cm}^{-2}$ (Rao \& Turnshek 2000).

According to Braun (1997), gas rich galaxies may contain a medium with $N_{H I}=4 \times$ $10^{22} \mathrm{~cm}^{-2}$. In Fig. 3 we show the scattering optical depth for this $\mathrm{H}$ I column density. For a higher column density system with $N_{H I}=4 \times 10^{22} \mathrm{~cm}^{-2}$, we obtain $\Delta \lambda=1.17 \AA$, which may result in underestimation of the redshift by $\Delta z=0.962 \times 10^{-3}$. This amounts to a velocity

shift of $\Delta v=289 \mathrm{~km} \mathrm{~s}^{-1}$, and may affect significantly the exact redshift determination of the absorbing system.

\section{Discussion}

Gavrila (1967) provided a quantum mechanical computation of the Rayleigh scattering cross section using the Green function in momentum space. Ferland (2001) obtained the power fit to the result of Gavrila (1967) and incorporated it in his photoionization code 'Cloudy,' in which the formula is useful for radiation with $\lambda>1410 \AA$ and for radiation nearer the Ly $\alpha$ line center Voigt function is proposed to be used. In the low energy limit where $\omega \ll \omega_{L y \alpha}$, the scattering cross section tends to be proportional to $\omega^{4}$, which is also expected from classical physics. This approximation is quite useful in dealing with basic radiative transfer, because the Voigt function (or equivalently the Lorentzian function) well 
approximates the true scattering cross section.

Therefore, in this work, we emphasize the asymmetric deviation of the true scattering cross section from the Lorentzian in frequency space, which may result in underestimation of the exact wavelength of the Ly $\alpha$ line center. Careful comparisons of redshifts determined using other lines may provide important clues to the kinematics of the absorbing systems. In particular, Prochaska \& Wolfe $(1997,1998)$ proposed that the absorption profiles observed in many DLA's are consistent with the hypothesis that they are formed in a rotating disk. The apparent line shift due to the asymmetric deviation around Ly $\alpha$ may affect the kinematic analysis of rotating galaxies.

Scattering of Ly $\alpha$ photons is also important in search for the first objects that are responsible for the reionization of the universe (Gunn \& Peterson 1965, Scheuer 1965). In several high red shift quasars with $z>6.2$ the presence of the Gunn-Peterson trough has been reported blueward of Ly $\alpha$ (Becker et al. 2001, Fan et al. 2003). The Gunn-Peterson optical depth will be important in the red wing part of Ly $\alpha$ (e.g. Miralda-Escudé 1998). In Eq. (13) the correction from the Lorentzian cross section is about $1 \%$ when we consider a width of the damped profile of about $2000 \mathrm{~km} \mathrm{~s}^{-1}$, for the characteristic expected value of the intergalactic medium (IGM) density at around $z=6$. Hence, the change in the cross section is equivalent to a change in the redshift of the Gunn-Peterson trough of about $1 \%$ of th ewidth of the damped profile or $20 \mathrm{~km} \mathrm{~s}^{-1}$, when only the first order term in $\Delta \omega / \omega_{21}$ is considered.

Another high column density media may be found in the atmospheres of supergiants, in which classical Rayleigh scattering is important (e.g. Isliker Nussbaumer \& Vogel 1989). Also about a half of the symbiotic stars believed to consist of a heavily mass losing giant and a white dwarf exhibit Raman scattered O VI features around $6830 \AA$ and $7088 \AA$ (Schmid 1989, Nussbaumer, Schmid, \& Vogel 1989). These features are originated from the resonance doublet O VI 1032, 1038 line photons that are scattered in a neutral region with $N_{H I} \sim 10^{22} \mathrm{~cm}^{-2}$. However, due to resonant scattering by neutral hydrogen in interstellar space, it will be difficult to accurately determine the asymmetry of the scattering cross section.

The author is very grateful to an anonymous referee for useful comments. This work is a result of research activities of the Astrophysical Research Center for the Structure and Evolution of the Cosmos (ARCSEC) funded by the Korea Science and Engineering Foundation. 


\section{REFERENCES}

Ahn, S. -H., Lee, H. -W. \& Lee, H. M., Journal of the Korean Astronomical Society, 2000, 33,29

Becker, R. H. et al. 2001, AJ, 122, 2850

Berestetskii,V.B., Lifshitz, E.M., \& Pitaevskii, L.P., 1971, Relativistic Quantum Mechanics, Pergamon Press

Bethe, H. A. \& Salpeter, E. E. 1967, Quantum Mechanics of One and Two Electron Atoms, Academic Press Inc., New York

Braun, R., 1997, ApJ, 484, 637

Fan, X. et al. 2003, AJ, 125, 1649

Ferland, G., 2001, Hazy, a brief introduction to Cloudy 94.00

Gavrila, M., 1967, Physical Review, 163, 147

Gunn, J. E., Peterson, B. A., 1965, ApJ, 142, 1633

Isliker, H., Nussbaumer, H., \& Vogel, M., 1989, A\& A, 219, 271

Lee, H. -W., \& Lee, K. W. 1997, MNRAS, 287, 211

Merzbacher, E. 1970, Quantum Mechanics, Wiley, New York

Miralda-Escudé, J., 1998, ApJ, 501, 15

Nussbaumer, H., Schmid, H. M.\& Vogel, M.,1989,A\& A, 221, L27

Peebles, P. J. E., 1993, Principles of Physical Cosmology, Princeton University Press, Princeton

Prochaska, J. X. \& Wolfe, A. M., 1997, ApJ, 487, 73

Prochaska, J. X. \& Wolfe, A. M., 1998, ApJ, 507, 113

Rao, S. M., Nestor, D. B., Turnshek, D. A., Lane, W. M., Monier, E. M., \& Bergeron, J., 2003, ApJ,

Rao, S. M., Turnshek, D. A., 2000, ApJS, 130, 1 
Sakurai, J. J., 1967, Advanced Quantum Mechanics, Addison-Wesley Publishing Company, Reading, Massachusetts

Saslow, W. M., Mills, D. L. 1969, Physical Review, 187, 1025

Scheuer, P. A. G. 1965, Nature, 207, 963

Schmid, H. M. 1989,A\& A, 211, L31

Turnshek, D. A., \& Rao, S., 1998, ApJ, 500, L115

This preprint was prepared with the AAS LATEX macros v5.0. 

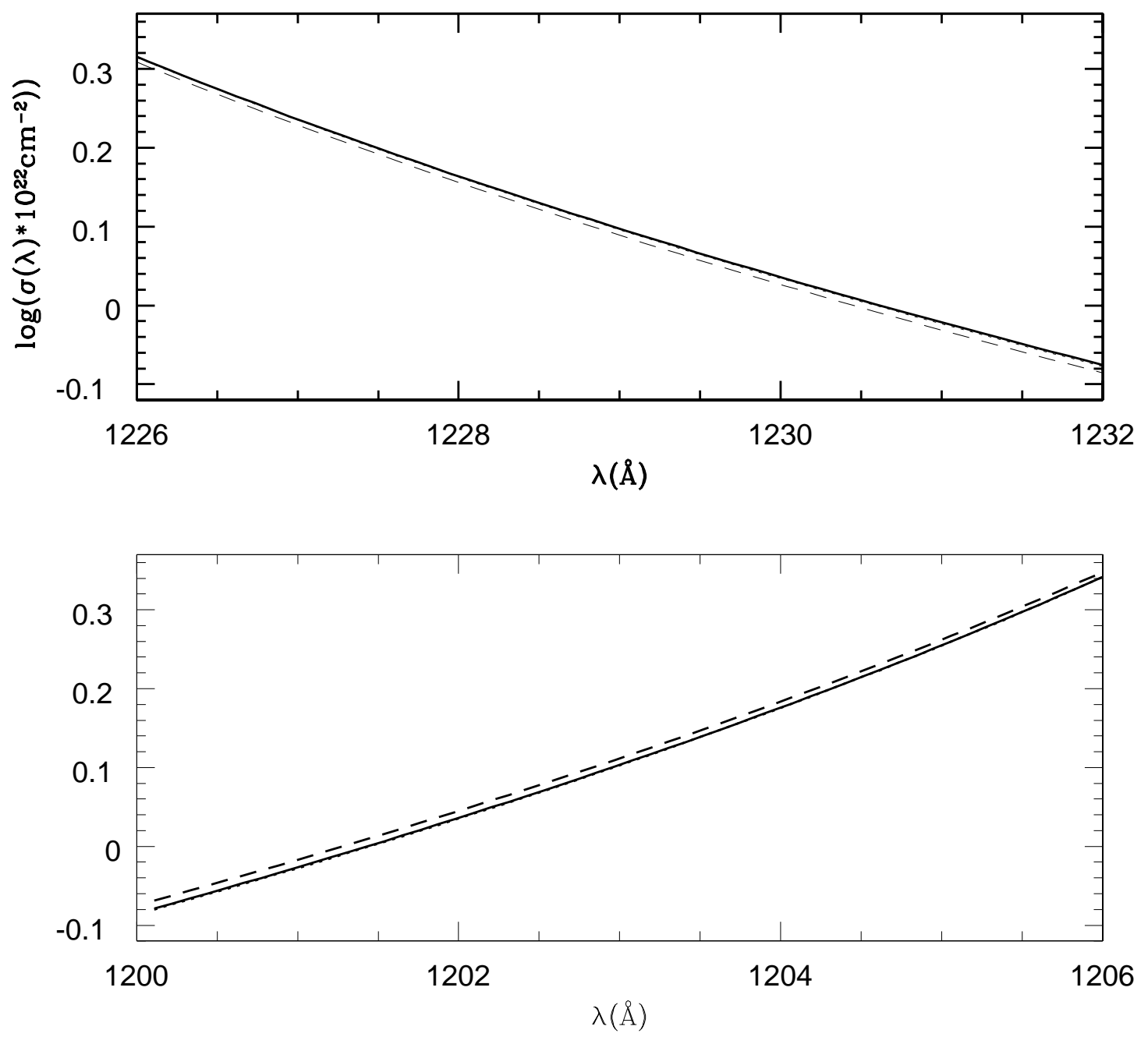

Fig. 1.- The scattering cross section around Ly $\alpha$. The result obtained from the KramersHeisenberg formula is shown by the solid line, the first order approximation to it obtained in Eq. 13 and the zeroth approximation that is proportional to $\Delta \omega^{-2}$ obtained from the Lorentzian by ignoring the damping part. The scattering cross section is smaller than the Lorentzian blueward of the line center, whereas it is larger in the opposite part. 


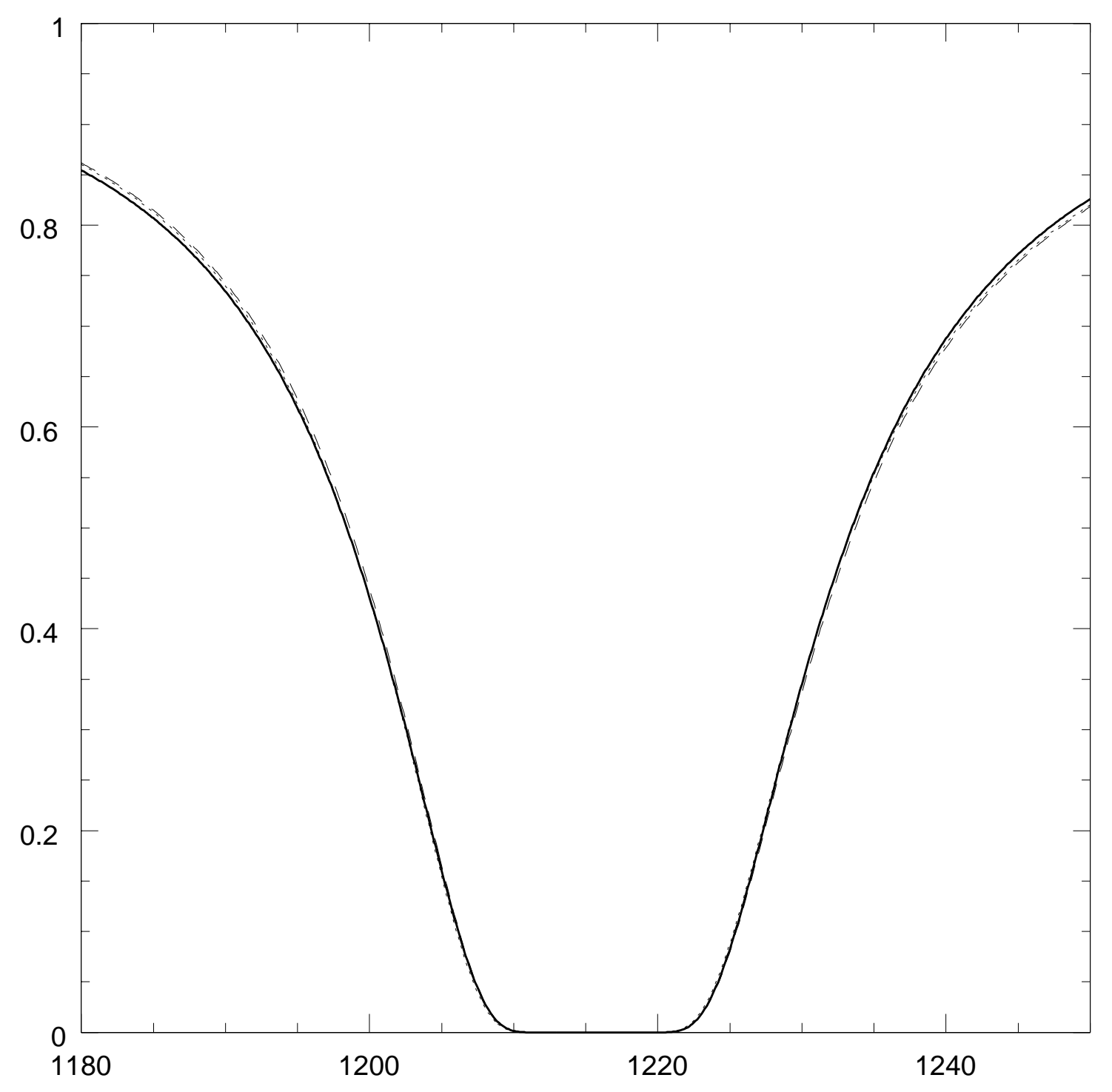

Fig. 2.- The absorption profile $e^{-\tau(\lambda)}$ expected from a damped Ly $\alpha$ system with a column density $N_{H I}=5 \times 10^{21} \mathrm{~cm}^{-2}$. The thick solid line represents the result obtained using the first-order corrected formula and the dotted line is the absorption profile obtained using the Voigt profile by shifting the center wavelength by an amount $\Delta \lambda=0.187 \AA$ to the blue part. The wavelength shift by this amount is determined using the least square method that best fits the thick solid line. 

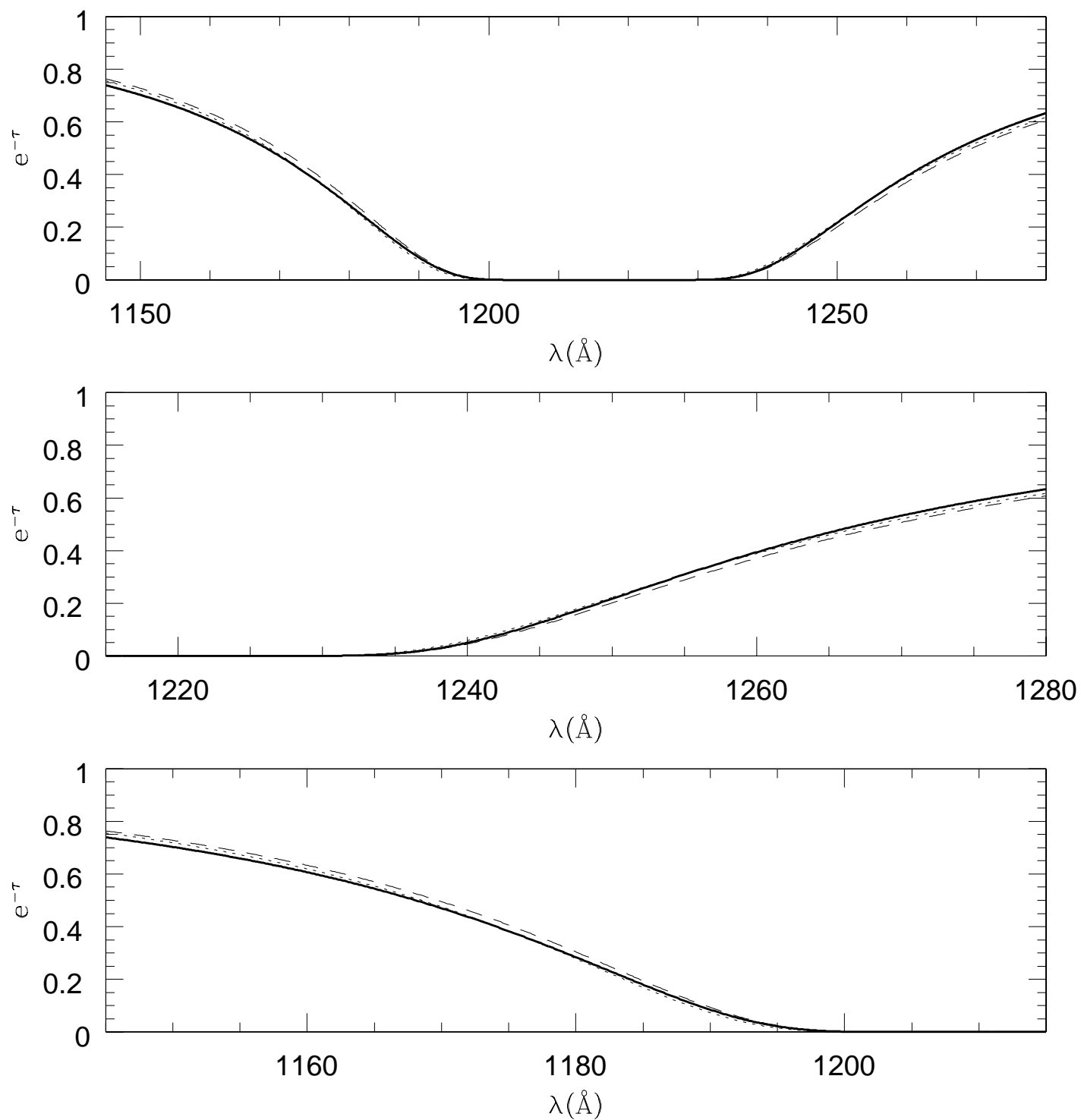

Fig. 3.- The absorption profile $e^{-\tau(\lambda)}$ expected from a damped Ly $\alpha$ system with a column density $N_{H I}=4 \times 10^{22} \mathrm{~cm}^{-2}$. The thick solid line represents the first-order correction. The best-fit wavelength shift from the Lorentzian fit is $\Delta \lambda=1.17 \AA$, which may result in underestimation of the redshift by $\Delta z=0.962 \times 10^{-3}$. 